\title{
嘧啶衍生物对牛蒡子苷元片段修饰的研究
}

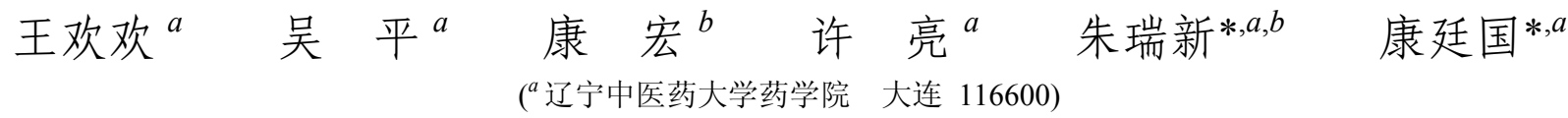 \\ $\left({ }^{b}\right.$ 同济大学生命科学与技术学院 上海 200092)
}

\begin{abstract}
摘要 用一系列具有生物活性的嘧啶衍生物修饰牛蒡子苷元, 旨在寻找增强牛蒡苷元子抗肿瘤活性的同时又能降低嘧 啶抗肿瘤副作用的先导化合物. 本研究通过把卤代后的嘧啶衍生物与牛蒡子苷元酚羟基相接, 合成得到 11 个新的化合 物, 通过 ${ }^{1} \mathrm{H}$ NMR 与 LC-MS 表征确定其结构. 最终, 增加了牛䓂子苷元抗肿瘤化合物库中化合物的数量, 为接下来的 体外活性篮选做准备.
\end{abstract}

关键词 牛蒡子苷元; 结构修饰; 嘧啶衍生物; 异分子孪生药物

\section{Modify a Fragment of Arctigenin with Pyrimidine Derivatives}

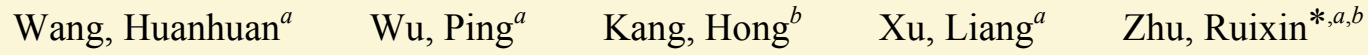 \\ Kang, Tingguo $*, a$ \\ ( ${ }^{a}$ School of Pharmacy, Liaoning University of Traditional Chinese Medicine, Dalian 116600) \\ ( ${ }^{b}$ School of Life Sciences and Technology, Tongji University, Shanghai 200092)
}

\begin{abstract}
In order to enhance the anti-tumor activity of the arctigenin and reduce the side effects of pyrimidine, arctigenin was modified with pyrimidine derivatives having biological activities. In this research, some halogenated pyrimidines were linked to the phenolic hydroxyl group of the arctigenin to gain eleven new compounds, and the structures of them were identified by LC-MS and ${ }^{1} \mathrm{H}$ NMR. So, the number of the anti-tumor compound libraries of compounds based on arctigenin was increased, and these compounds were prepared for the in-vitro activity screening in the near future. By our work, when enhance the alkalinity and increase the water content in DMF, the etherification reaction is promoted, to a certain extent.

Keywords arctigenin; structural modification; pyrimidine derivatives; nonidentical twin drug
\end{abstract}

当今社会, 肿瘤的发病越来越多, 而肿瘤的治疗药 物的开发日渐得到重视, 通过篮选具有抗肿瘤活性的天 然植物, 选择其中抗肿瘤作用明显, 副作用小, 可以量 产的天然药物, 提取其有效的抗肿瘤成分, 用有机合成 的方法对其某个片段进行修饰, 以期减毒增效, 不失为 一条抗肿瘤药物开发的路径.

牛蒡(aictium lappa L.), 菊科两年生草本植物, 其干 燥成熟果实牛蒡子, 味辛、苦、寒, 有疏散风热、解毒 透疹、利咽消肿等功效, 为常用中药. 其主要活性成分 为木脂素及其苷类, 牛蒡子苷元(arctigenin)为其中的二 芐基丁内酯型木脂素类化合物. 现代药理表明牛蒡子苷 元具有抗病毒、抗肿瘤、诱导白血病细胞调亡以及调节 免疫活性的作用 ${ }^{[1]}$.
嘧啶是一类带碱性有一个碳氮环的含氮化合物, 是 人类遗传物质的重要组成部分, 具有一定的生物活性. 5-氟尿嘧啶、二喃氟啶、呋喃氟尿嘧啶、阿糖胞苷、氟 豚苷等均具有抗肿瘤的药理作用.

虽然二者都有抗肿瘤活性. 但相比较而言, 嘧啶类 效果明显, 副作用大, 对人体伤害严重, 而牛蒡子苷元 抗肿瘤效果相对偏弱.

将两个药效结构单位组合成新的分子的原理已经 在多个上市药物中体现，如双阿司匹林和水杨酸与扑热 息痛结合而成的 Acetaminosalol. 理论上讲, 异分子孪 生药物在一定程度上存在协同作用, 同时相对降低了药 物与作用靶点的亲和力, 故而, 可以起到增效减毒的作 用. 本研究即根据此理论展开. 将均具有抗肿瘤作用的

*E-mail: rxzhu@tongji.edu.cn

Received March 19, 2012; revised May 29, 2012; published online June 6, 2012.

Project supported by the National Natural Science Foundation of China (No. 30976611), the Research Fund for the Doctoral Program of Higher Education of China (No. 20100072120050) and the Traditional Chinese Medicine Modernization of Shanghai (No. 09dZ1972800).

国家自然科学基金(No. 30976611)、中国高等教育博士研究基金计划(No. 20100072120050)和上海中药现代化(No. 09dZ1972800)资助项目. 
牛蒡子苷元和嘧啶衍生物通过醚键结合, 形成异分子孪 生化合物, 有望增强牛蒡子苷元抗肿瘤活性的同时降低 嘧啶衍生物的毒副作用.

\section{1 结果与讨论}

选择合适的起始原料, 利用 Schemes 1, 2 所示方法 经过一步或多步反应, 合成出 9 种嘧啶衍生物; 通过 Sandmeyer 反应将嘧啶环上的氨基溴取代得到 $9 a$ 和 $9 b$ (Eq. 1). 然而, 如表 1 所示, 9a 和 $9 \mathbf{b}$ 的产率均非常低, 结 合本实验室经验, 嘧啶、喹啉等杂环类化合物的氨基利 用 Sandmeyer 反应的条件难以较好地转化成氯或者溴. 关于此类问题的后续研究正在进行中.

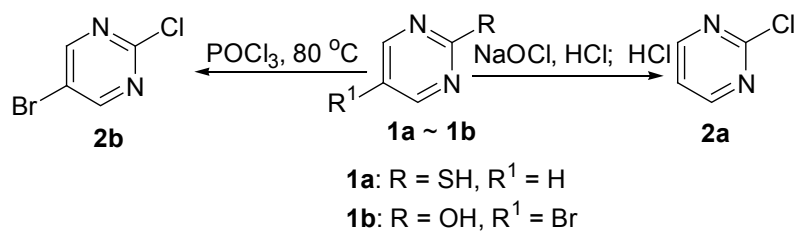

Scheme 1
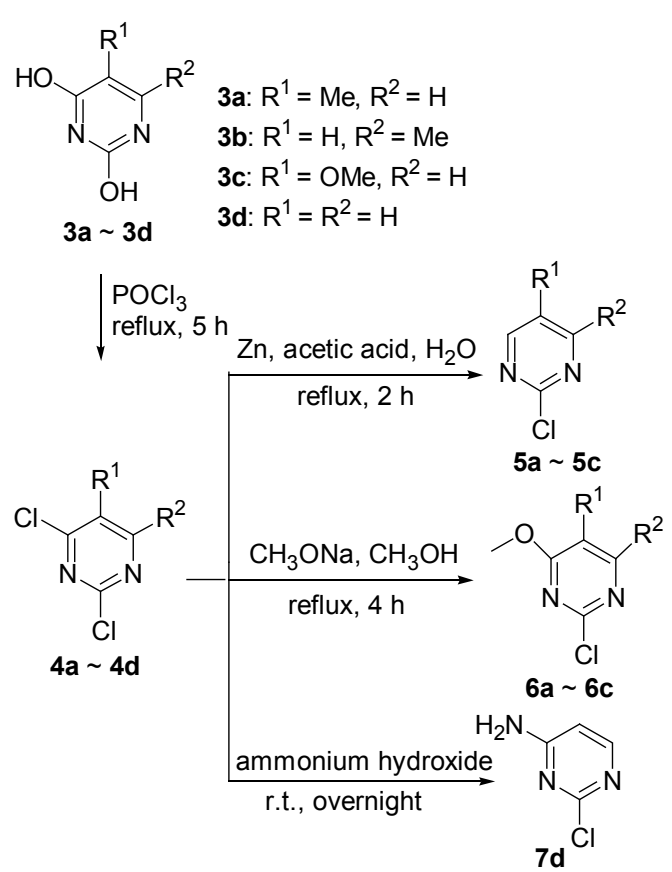

Scheme 2

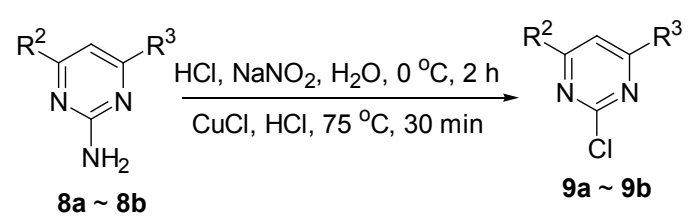

8a: $\mathrm{R}^{2}=\mathrm{CF}_{3}, \mathrm{R}^{3}=\mathrm{H}$

8b: $R^{2}=R^{3}=O M e$
表 1 嘧啶衍生物的合成

Table 1 Synthesis of the pyrimidine derivatives

\begin{tabular}{cccccccc}
\hline Entry & Substrate & $\mathrm{R}$ & $\mathrm{R}^{1}$ & $\mathrm{R}^{2}$ & $\mathrm{R}^{3}$ & Product & Yield/\% \\
\hline 1 & 1a & $\mathrm{SH}$ & $\mathrm{H}$ & $\mathrm{H}$ & $\mathrm{H}$ & $\mathbf{2 a}$ & 70.0 \\
2 & 1b & $\mathrm{OH}$ & $\mathrm{Br}$ & $\mathrm{H}$ & $\mathrm{H}$ & $\mathbf{2 b}$ & 62.0 \\
3 & 3a & $\mathrm{OH}$ & $\mathrm{Me}$ & $\mathrm{H}$ & $\mathrm{OH}$ & $\mathbf{5 a}$ & 60.7 \\
4 & 3a & $\mathrm{OH}$ & $\mathrm{Me}$ & $\mathrm{H}$ & $\mathrm{OH}$ & $\mathbf{6 a}$ & 57.8 \\
5 & 3b & $\mathrm{OH}$ & $\mathrm{H}$ & $\mathrm{Me}$ & $\mathrm{OH}$ & $\mathbf{5 b}$ & 39.2 \\
6 & 3b & $\mathrm{OH}$ & $\mathrm{H}$ & $\mathrm{Me}$ & $\mathrm{OH}$ & $\mathbf{6 b}$ & 63.3 \\
7 & $\mathbf{3 c}$ & $\mathrm{OH}$ & $\mathrm{OMe}$ & $\mathrm{H}$ & $\mathrm{OH}$ & $\mathbf{5 c}$ & 57.4 \\
8 & $\mathbf{3 c}$ & $\mathrm{OH}$ & $\mathrm{OMe}$ & $\mathrm{H}$ & $\mathrm{OH}$ & $\mathbf{6 c}$ & 60.8 \\
9 & $\mathbf{3 d}$ & $\mathrm{OH}$ & $\mathrm{H}$ & $\mathrm{H}$ & $\mathrm{OH}$ & $\mathbf{7 d}$ & 50.0 \\
10 & $\mathbf{8 a}$ & $\mathrm{NH}_{2}$ & $\mathrm{H}$ & $\mathrm{CF}$ & $\mathrm{H}$ & $\mathbf{9 a}$ & 18.4 \\
11 & $\mathbf{8 b}$ & $\mathrm{NH}_{2}$ & $\mathrm{H}$ & $\mathrm{OMe}$ & $\mathrm{OMe}$ & $\mathbf{9 b}$ & 20.0 \\
\hline
\end{tabular}

将牛蒡子苷元与嘧啶衍生物通过醚键结合, 合成得 到 11 种新的牛蒡子苷元修饰物(Eq. 2, 表 2).<smiles>[R]C1CCC(=C)C1Cc1ccc(OC)c(OC)c1</smiles>

本研究中, 在将嘧啶衍生物与牛蒡子苷元偶合的时 候，曾尝试碳酸钠，发现产率不如使用碳酸钾高; 此外, 经对比, 在反应液中加入 1 滴水后, 反应速率有所提高 (TLC 监测), 很可能是因为水的加入增加了反应体系中 碳酸钾的溶解度, 从而使体系碱性增强, 促进反应的进 行. 不足的是, 未能研究微量水的加入能否对产率的提 高做出贡献. 理论上讲, 虽然少量水的加入能够提高反 应速率，但是，另一方面，由于醚化反应生成水，而水 含量增加会在一定程度上抑制反应的进行. 这一点可以 从醚化反应产率一直维持在 $60 \%$ 左右得到证实. 若此, 是不是如果能够及时有效除去反应生成的水, 产率会有 
表 2 嘧啶衍生物-牛蒡子苷元醚的合成

Table 2 Synthesis of the pyrimidine-derivatives-arctigenin ether

\begin{tabular}{ccccccc}
\hline Entry & Substrate & $\mathrm{R}^{1}$ & $\mathrm{R}^{2}$ & $\mathrm{R}^{3}$ & Product & Yield/\% \\
\hline 1 & $\mathbf{2 a}$ & $\mathrm{H}$ & $\mathrm{H}$ & $\mathrm{H}$ & $\mathbf{1 1 a}$ & 62.0 \\
2 & $\mathbf{2 b}$ & $\mathrm{Br}$ & $\mathrm{H}$ & $\mathrm{H}$ & $\mathbf{1 1 b}$ & 56.0 \\
3 & $\mathbf{5 a}$ & $\mathrm{Me}$ & $\mathrm{H}$ & $\mathrm{H}$ & $\mathbf{1 1 c}$ & 60.0 \\
4 & $\mathbf{6 a}$ & $\mathrm{Me}$ & $\mathrm{H}$ & $\mathrm{OMe}$ & $\mathbf{1 1 d}$ & 60.3 \\
5 & $\mathbf{5 b}$ & $\mathrm{H}$ & $\mathrm{Me}$ & $\mathrm{H}$ & $\mathbf{1 1 e}$ & 48.0 \\
6 & $\mathbf{6 b}$ & $\mathrm{H}$ & $\mathrm{Me}$ & $\mathrm{OMe}$ & $\mathbf{1 1 f}$ & 67.8 \\
7 & $\mathbf{5 c}$ & $\mathrm{OMe}$ & $\mathrm{H}$ & $\mathrm{H}$ & $\mathbf{1 1 g}$ & 50.0 \\
8 & $\mathbf{6 c}$ & $\mathrm{OMe}$ & $\mathrm{H}$ & $\mathrm{OMe}$ & $\mathbf{1 1 h}$ & 58.9 \\
9 & $\mathbf{7 d}$ & $\mathrm{H}$ & $\mathrm{H}$ & $\mathrm{NH}_{2}$ & $\mathbf{1 1 i}$ & 40.0 \\
10 & $\mathbf{9 a}$ & $\mathrm{H}$ & $\mathrm{CF}$ & $\mathrm{H}$ & $\mathbf{1 1 j}$ & 75.0 \\
11 & $\mathbf{9 b}$ & $\mathrm{H}$ & $\mathrm{OMe}_{3}$ & $\mathrm{OMe}$ & $\mathbf{1 1 k}$ & 66.3 \\
\hline
\end{tabular}

所提高呢? 带着这个假设, 本研究小组下一步工作将在 继续对牛蒡子苷元结构修饰的同时, 对 DMF 中水含量 对醚化反应的影响做进一步研究.

\section{2 结论}

总计合成出嘧啶衍生物-牛蒡子苷元醚系列化合物 11 个, 扩充了牛蒡子苷元修饰物库, 为不久即将展开的 体外活性篮选做好前期准备.

\section{3 实验部分}

\section{1 仪器与试剂}

牛蒡子苷元来源于辽宁中医药大学药学院辽宁省 中药鉴定与品质评价重点实验室; 实验所用化学试剂全 部购自国药集团化学试剂有限公司, 分析纯; 薄层层析 硅胶板购自上海上邦实业有限公司 GF254.

试验中用到的主要仪器有: 瑞士 AVANCE 500 MHz 型核磁共振仪, Agilent 6100 型 LC-MS, UV-1700 型 紫外可见分光光度计 (岛津), 旋转蒸发仪(RE-52AA, 上 海亚荣生化仪器厂).

\section{2 实验方法}

\subsection{1 $2 \mathrm{a}, \mathbf{2 b}$ 和 $7 \mathbf{d}$ 的合成}

参照文献[2]合成化合物 $\mathbf{2 a}$ 和 $\mathbf{2 b}$, 参照文献[3]合成 化合物 7d.

\subsection{2 以 $\mathbf{5 b}$ 为例合成 $\mathbf{5 a} \sim \mathbf{5 c}$}

将 2,4-二羟基-6-甲基嘧啶 $(\mathbf{3 b}, 50.0 \mathrm{~g}, 0.4 \mathrm{~mol}$ )缓慢 加到 $\mathrm{POCl}_{3}(200.0 \mathrm{~g}, 1.3 \mathrm{~mol})$ 中, 边加边搅拌, 加毕, 油 浴回流 $5 \mathrm{~h}$. 反应结束后, 降至室温, 减压除去大部分 $\mathrm{POCl}_{3}$, 剩余物缓慢倒入冰水混合液 $(250 \mathrm{~g}$ 冰, $250 \mathrm{~mL}$ 水)中, 搅拌下加入 $\mathrm{NaHCO}_{3}$ 固体至水溶液中性, 乙酸乙 酯萃取水溶液, 乙酸乙酯层用无水硫酸镁干燥, 减压浓 缩得棕色液体, 乙酸乙酯-石油醚 $(V: V=1: 10)$ 重结
晶, 得 $58 \mathrm{~g}$ 棕色固体 $\mathbf{4 b}$, 产率 $90 \%$.

将 $4 \mathrm{~b}(58.0 \mathrm{~g}, 0.36 \mathrm{~mol})$ 置于 $1 \mathrm{~L}$ 三颈瓶中, 加入水 $(200 \mathrm{~mL})$ 和冰醋酸 $(20 \mathrm{~mL})$, 搅拌混匀, 体系加热至 65 ${ }^{\circ} \mathrm{C}$, 分批加入锌粉 $(35.0 \mathrm{~g}, 0.54 \mathrm{~mol}, 1.5$ equiv.)加毕, 保 温反应 $4 \mathrm{~h}$. 反应结束后反应液温度冷却至室温, 抽滤, 滤液用乙酸乙酯萃取 $(200 \mathrm{~mL} \times 3)$, 合并有机相, 并用无 水硫酸镁干燥, 抽滤, 滤液减压蒸干, 得淡黄色粗品, 乙酸乙酯重结晶得 $20.0 \mathrm{~g}$ 白色固体 $\mathbf{5 b}$, 产率 $44 \%$.

\section{2 .3 以 $6 \mathrm{~b}$ 为例合成 $6 \mathrm{a} \sim 6 \mathrm{c}$}

取棕色固体 4b $(10.0 \mathrm{~g}, 0.06 \mathrm{~mol})$ 与甲醇钠 $(3.2 \mathrm{~g}$, $0.06 \mathrm{~mol}$ )在甲醇 $(100 \mathrm{~mL})$ 中搅拌回流 $4 \mathrm{~h}$, 冷却至室温, 抽滤, 减压除去甲醇, 加入乙酸乙酯 $100 \mathrm{~mL}$, 有机相水 洗后用无水硫酸镁干燥, 抽滤, 滤液减压蒸干得 $6.0 \mathrm{~g}$ 无 色液体 $6 \mathbf{b}$, 产率 $63.5 \%$.

\subsection{4 以 $9 \mathrm{~b}$ 为例合成 $9 \mathrm{a} \sim 9 \mathrm{~b}$}

将 $8 \mathbf{b}(0.68 \mathrm{~g}, 4.6 \mathrm{mmol})$ 溶于 $\mathrm{HCl}(38 \%, 3.0 \mathrm{~mL})$, 冰 浴搅拌 $0.5 \mathrm{~h}$. 缓慢滴加 $2.5 \mathrm{~mol} / \mathrm{L}$ 亚硝酸钠水溶液 $(2.0$ $\mathrm{mL}, 5.1 \mathrm{mmol}, 1.1$ equiv.). 滴加完毕, 将重氮盐溶液滴 加到事先预热到 $75{ }^{\circ} \mathrm{C}$ 的 $\mathrm{CuCl}(0.54 \mathrm{~g}, 5.5 \mathrm{mmol}, 1.2$ equiv.)的 $\mathrm{HCl}(38 \%, 3 \mathrm{~mL})$ 的溶液中, 反应 $30 \mathrm{~min}$ 后冷 却至室温, 用 $8 \mathrm{~mol} / \mathrm{L} \mathrm{NaOH}$ 水溶液调 $\mathrm{pH}$ 至 10 , 二氯甲 烷萃取三次 $(10 \mathrm{~mL} \times 3)$, 有机相用饱和氯化钠水溶液洗 一次, 水洗一次, 无水硫酸镁干燥后, 减压浓缩, 硅胶 柱层析分离纯化 $[V($ 乙酸乙酯 $): V($ 石油醚 $)=1: 5 \sim 1:$ $1]$. 得 $0.19 \mathrm{~g}$ 白色晶体 $\mathbf{9 b}$, 产率 $20.0 \%$. ${ }^{1} \mathrm{H} \mathrm{NMR}\left(\mathrm{CDCl}_{3}\right.$, $500 \mathrm{MHz}) \delta$ : $5.98(\mathrm{~s}, 1 \mathrm{H}), 3.97$ (s, 6H).

2-氯-4-三氟甲基嘧啶 (9a): 无色透明液体, 产率 18.4\%. ${ }^{1} \mathrm{H}$ NMR $\left(\mathrm{CDCl}_{3}, 500 \mathrm{MHz}\right) \delta: 8.94(\mathrm{~d}, J=4.5 \mathrm{~Hz}$, $1 \mathrm{H}), 7.65(\mathrm{~d}, J=4.5 \mathrm{~Hz}, 6 \mathrm{H})$.

\subsection{5 以 $11 \mathrm{c}$ 为例合成化合物 $11 \mathrm{a} \sim 11 \mathrm{k}$}

于 $25 \mathrm{~mL}$ 圆底烧瓶中, 依次加入牛蒡子苷元 $(0.2 \mathrm{~g}$, $0.54 \mathrm{mmol}), 2$-氯-5-甲基嘧啶 $(0.08 \mathrm{~g}, 0.65 \mathrm{mmol}, 1.2$ equiv.), 碳酸钾 $(0.08 \mathrm{~g}, 0.58 \mathrm{mmol}, 1.1$ equiv.), DMF $(8 \mathrm{~mL})$, 纯净水 1 滴, 油浴 $150{ }^{\circ} \mathrm{C}$ 加热过夜. 体系冷却至 室温后向其中加入 $20 \mathrm{~mL}$ 水, 用二氯甲烷 $(30 \mathrm{~mL} \times 4)$ 萃 取, 合并有机相, 并用饱和氯化钠水溶液洗涤两次; 有 机相用无水硫酸镁干燥后抽滤, 滤液减压蒸干, 得棕色 液体, 过硅胶柱 $[V($ 乙酸乙酯 $): V($ 石油醚 $)=1: 1]$, 得 $0.15 \mathrm{~g}$ 浅棕色油状物 (3R,4R)-3-[3-甲氧基-4-(2-O-5-甲 基-嘧啶基)-苯甲基]-4-(3,4-二甲氧基-苯甲基)-丁内酯 (11c), 产率 60\%. ${ }^{1} \mathrm{H} \mathrm{NMR}\left(\mathrm{CDCl}_{3}, 500 \mathrm{MHz}\right) \delta: 8.32(\mathrm{~s}$, 2H), $7.09(\mathrm{~d}, J=8.0 \mathrm{~Hz}, 1 \mathrm{H}), 6.80(\mathrm{~d}, J=1.8 \mathrm{~Hz}, 1 \mathrm{H})$, $6.78 \sim 6.72(\mathrm{~m}, 1 \mathrm{H}), 6.55(\mathrm{dd}, J=8.1,1.9 \mathrm{~Hz}, 2 \mathrm{H}), 6.51(\mathrm{~d}$, $J=1.8 \mathrm{~Hz}, 1 \mathrm{H}), 4.19(\mathrm{dd}, J=9.1,7.0 \mathrm{~Hz}, 1 \mathrm{H}), 3.91(\mathrm{dd}$, $J=9.1,7.4 \mathrm{~Hz}, 1 \mathrm{H}), 3.85$ (s, 3H), 3.82 (s, 3H), 3.68 (s, 3H), 
3.04 (dd, $J=14.1,5.3 \mathrm{~Hz}, 1 \mathrm{H}$ ), 2.97 (dd, $J=14.1,6.8 \mathrm{~Hz}$, $2 \mathrm{H}$ ), $2.71 \sim 2.49$ (m, 4H, 7, 8 \& $8^{\prime}$ ), 2.2 (s, 3H); ESI-MS $m / z: 465[\mathrm{M}+1]^{+}$.

(3R,4R)-3-[3- 甲氧基-4-(2- $O$ - 嘧啶基)-苯甲基]4-(3,4-二甲氧基-苯甲基)-丁内酯(11a): 反应参照 $11 \mathrm{c}$, 但后处理时加入 $20 \mathrm{~mL}$ 水, 有白色固体析出, 抽滤, 滤 饼依次用纯净水、石油醚洗涤, 固体红外灯下干燥, 得 $0.15 \mathrm{~g}$ 白色固体, 产率 $62 \% .{ }^{1} \mathrm{H}$ NMR $\left(\mathrm{CDCl}_{3}, 500 \mathrm{MHz}\right)$ $\delta: 8.51$ (d, $J=4.8 \mathrm{~Hz}, 2 \mathrm{H}, 4 " \&$ 6"), $7.11(\mathrm{~d}, J=8.0 \mathrm{~Hz}$, $\left.1 \mathrm{H}, 6^{\prime}\right), 7.00$ (t, $\left.J=4.8 \mathrm{~Hz}, 1 \mathrm{H}\right), 6.81$ (d, $\left.J=1.5 \mathrm{~Hz}, 1 \mathrm{H}\right)$, $6.76(\mathrm{~d}, J=8.1 \mathrm{~Hz}, 2 \mathrm{H}), 6.57 \sim 6.53(\mathrm{~m}, 1 \mathrm{H}), 6.52(\mathrm{~s}, 1 \mathrm{H})$, 4.19 (dd, $J=8.9,7.1 \mathrm{~Hz}, 1 \mathrm{H}), 3.94 \sim 3.89(\mathrm{~m}, 1 \mathrm{H}), 3.85$ (s, $3 \mathrm{H}), 3.82$ (s, 3H), 3.69 (s, 3H), 3.05 (dd, $J=14.1,5.3 \mathrm{~Hz}$, $1 \mathrm{H}), 3.01 \sim 2.96(\mathrm{~m}, 1 \mathrm{H}), 2.70 \sim 2.52(\mathrm{~m}, 4 \mathrm{H})$; ESI-MS $m / z: 451[\mathrm{M}+1]^{+}$.

(3R,4R)-3-[4-(2-O-5-溴-嘧啶基)-3- 甲氧基-苯甲 基]-4-(3,4-二甲氧基-苯甲基)-丁内酯(11b): $0.18 \mathrm{~g}$ 棕黄 色固体, 产率 56\%. ${ }^{1} \mathrm{H} \mathrm{NMR}\left(\mathrm{CDCl}_{3}, 500 \mathrm{MHz}\right) \delta: 8.51(\mathrm{~s}$, 2H), $7.08(\mathrm{~d}, J=8.0 \mathrm{~Hz}, 1 \mathrm{H}), 6.80(\mathrm{~d}, J=1.5 \mathrm{~Hz}, 1 \mathrm{H}), 6.75$ (dd, $J=10.8,4.8 \mathrm{~Hz}, 2 \mathrm{H}), 6.57 \sim 6.53(\mathrm{~m}, 1 \mathrm{H}), 6.51(\mathrm{~d}$, $J=1.6 \mathrm{~Hz}, 1 \mathrm{H}), 4.19(\mathrm{dd}, J=9.0,7.3 \mathrm{~Hz}, 1 \mathrm{H}), 3.95 \sim 3.88$ (m, 1H), 3.85 (s, 3H), $3.82(\mathrm{~s}, 3 \mathrm{H}), 3.69(\mathrm{~s}, 3 \mathrm{H}), 3.03$ (dd, $J=14.0,5.5 \mathrm{~Hz}), 2.98(\mathrm{t}, J=7.1 \mathrm{~Hz}, 1 \mathrm{H}), 2.69 \sim 2.48(\mathrm{~m}$, $4 \mathrm{H})$; ESI-MS $m / z: 529[\mathrm{M}+1]^{+}$.

(3R,4R)-3-[3-甲氧基-4-(2-O-4-甲基-嘧啶基)-苯甲 基]-4-(3,4-二甲氧基-苯甲基)-丁内酯(11e): $0.12 \mathrm{~g}$ 棕红 色液体, 产率 $48 \% .{ }^{1} \mathrm{H}$ NMR $\left(\mathrm{CDCl}_{3}, 500 \mathrm{MHz}\right) \delta: 7.39$ (s, 1H), 7.05 (d, $J=8.0 \mathrm{~Hz}, 1 \mathrm{H}), 6.99(\mathrm{~s}, 1 \mathrm{H}), 6.80$ (d, $J=$ $1.6 \mathrm{~Hz}, 1 \mathrm{H}), 6.78 \sim 6.72(\mathrm{~m}, 2 \mathrm{H}), 6.57(\mathrm{dd}, J=8.1,1.7 \mathrm{~Hz}$, $1 \mathrm{H}), 6.51(\mathrm{~d}, J=1.7 \mathrm{~Hz}, 1 \mathrm{H}), 4.18(\mathrm{dd}, J=9.0,7.4 \mathrm{~Hz}$, $1 \mathrm{H}), 3.90$ (dd, $J=15.4,6.6 \mathrm{~Hz}, 1 \mathrm{H}), 3.85$ (s, 3H), 3.82 (s, $3 \mathrm{H}), 3.72(\mathrm{~s}, 3 \mathrm{H}), 3.05 \sim 2.93(\mathrm{~m}, 1 \mathrm{H}), 2.72 \sim 2.48(\mathrm{~m}$, 1H), 1.99(s, 3H); ESI-MS $m / z: 465[\mathrm{M}+1]^{+}$.

(3R,4R)-3-[3-甲氧基-4-(2-O-4-甲氧基-5-甲基-嘧啶 基)-苯甲基]-4-(3,4-二甲氧基-苯甲基)-丁内酯(11d): $0.16 \mathrm{~g}$ 无色透明液体, 产率 $60.3 \%,{ }^{1} \mathrm{H} \mathrm{NMR}\left(\mathrm{CDCl}_{3}, 500\right.$ MHz) $\delta: 8.27$ (d, $J=5.0 \mathrm{~Hz}, 1 \mathrm{H}), 7.09$ (d, $J=8.0 \mathrm{~Hz}, 1 \mathrm{H})$, $6.85(\mathrm{~d}, J=5.0 \mathrm{~Hz}, 1 \mathrm{H}), 6.80(\mathrm{~d}, J=1.7 \mathrm{~Hz}, 1 \mathrm{H}), 6.77 \sim$ $6.72(\mathrm{~m}, 1 \mathrm{H}), 6.56 \sim 6.52(\mathrm{~m}, 1 \mathrm{H}), 6.51(\mathrm{~d}, J=1.7 \mathrm{~Hz}$, $1 \mathrm{H}), 5.29(\mathrm{~s}, 3 \mathrm{H}), 4.19$ (dd, $J=9.0,7.0 \mathrm{~Hz}), 3.91$ (dd, $J=$ 9.1, $7.4 \mathrm{~Hz}), 3.84$ (s, 3H), 3.81 (s, 3H), 3.69 (s, 3H), 3.01 (dd, $J=14.1,6.1 \mathrm{~Hz}, 2 \mathrm{H}), 2.70 \sim 2.52(\mathrm{~m}, 4 \mathrm{H}), 2.46$ (s, $3 \mathrm{H}, 5$ "- $\left.\mathrm{CH}_{3}\right)$; ESI-MS $m / z$ : $495[\mathrm{M}+1]^{+}$.

(3R,4R)-3-[3-甲氧基-4-(2-O-4-甲氧基-6-甲基-嘧啶 基)-苯甲基]-4-(3,4-二甲氧基-苯甲基)-丁内酯(11f):
$0.18 \mathrm{~g}$ 淡黄色粉末, 产率 67.8\%. ${ }^{1} \mathrm{H}$ NMR $\left(\mathrm{CDCl}_{3}, 500\right.$ MHz) $\delta: 7.10(\mathrm{~d}, J=8.0 \mathrm{~Hz}, 1 \mathrm{H}), 6.82(\mathrm{~d}, J=1.5 \mathrm{~Hz}, 1 \mathrm{H})$, $6.76(\mathrm{~d}, J=8.1 \mathrm{~Hz}, 2 \mathrm{H}), 6.56 \sim 6.53(\mathrm{~m}, 1 \mathrm{H}), 6.51(\mathrm{~s}, 1 \mathrm{H})$, $5.78(\mathrm{~s}, 1 \mathrm{H}) 4.19(\mathrm{dd}, J=8.9,7.1 \mathrm{~Hz}, 1 \mathrm{H}), 3.94 \sim 3.89(\mathrm{~m}$, $1 \mathrm{H}), 3.84(\mathrm{~s}, 3 \mathrm{H}), 3.83$ (s, 3H), 3.69 (s, 3H), 3.08 2.95 (m, 2H), $3.04(\mathrm{~s}, 3 \mathrm{H}), 2.84(\mathrm{~s}, 3 \mathrm{H}), 2.70 \sim 2.52(\mathrm{~m}, 4 \mathrm{H})$; ESI-MS $m / z: 495[\mathrm{M}+1]^{+}$.

(3R,4R)-3-[3-甲氧基-4-(2-O-5-甲氧基-嘧啶基)-苯 甲基]-4-(3,4-二甲氧基-苯甲基)-丁内酯(11g)：0.13 g 橙 黄色油状液体, 产率 $50 \% .{ }^{1} \mathrm{H} \mathrm{NMR}\left(\mathrm{CDCl}_{3}, 500 \mathrm{MHz}\right) \delta$ : $8.16(\mathrm{~s}, 2 \mathrm{H}), 7.08(\mathrm{~d}, J=8.0 \mathrm{~Hz}, 1 \mathrm{H}), 6.79(\mathrm{~d}, J=2.0 \mathrm{~Hz}$, $1 \mathrm{H}), 6.76 \sim 6.72(\mathrm{~m}, 2 \mathrm{H}), 6.55 \sim 6.50(\mathrm{~m}, 4 \mathrm{H}), 3.90(\mathrm{dd}$, $J=9.0,16.5 \mathrm{~Hz}, 1 \mathrm{H}), 3.84(\mathrm{~s}, 6 \mathrm{H}), 3.81(\mathrm{~s}, 3 \mathrm{H}), 3.68(\mathrm{~s}$, $3 \mathrm{H}), 3.64(\mathrm{t}, J=6.5 \mathrm{~Hz}, 1 \mathrm{H}), 3.05 \sim 2.94(\mathrm{~m}, 2 \mathrm{H}), 2.67 \sim$ $2.51(\mathrm{~m}, 4 \mathrm{H})$; ESI-MS $m / z$ : $481[\mathrm{M}+1]^{+}$.

(3R,4R)-3-[3-甲氧基-4-(2- $O-4,5$-二甲氧基-嘧啶基)苯甲基]-4-(3,4-二甲氧基-苯甲基)-丁内酯(11h): $0.16 \mathrm{~g}$ 棕色固体, 产率 58.9\%. ${ }^{1} \mathrm{H}$ NMR $\left(\mathrm{CDCl}_{3}, 500 \mathrm{MHz}\right) \delta$ : 8.16 (s, 1H), 7.08 (d, $J=8.0 \mathrm{~Hz}, 1 \mathrm{H}), 6.79$ (d, $J=2.0 \mathrm{~Hz}$, $1 \mathrm{H},), 6.76 \sim 6.72(\mathrm{~m}, 2 \mathrm{H}), 6.55 \sim 6.50(\mathrm{~m}, 4 \mathrm{H}), 3.90(\mathrm{dd}$, $J=9.0,16.5 \mathrm{~Hz}, 1 \mathrm{H}), 3.84(\mathrm{~s}, 6 \mathrm{H}), 3.81(\mathrm{~s}, 3 \mathrm{H}), 3.68$ (s, $3 \mathrm{H}), 3.68(\mathrm{~s}, 3 \mathrm{H}), 3.64(\mathrm{t}, J=6.5 \mathrm{~Hz}, 1 \mathrm{H}), 3.05 \sim 2.94(\mathrm{~m}$, $2 \mathrm{H}), 2.67 \sim 2.51(\mathrm{~m}, 4 \mathrm{H})$; ESI-MS $m / z: 511[\mathrm{M}+1]^{+}$.

$(3 R, 4 R)$-3-[3-甲氧基-4-(2-O-4-氨基-嘧啶基)-苯甲 基]-4-(3,4-二甲氧基-苯甲基)-丁内酯(11i): $0.1 \mathrm{~g}$ 黄褐色 粉末, 产率 40\%. ${ }^{1} \mathrm{H}$ NMR (DMSO, $\left.500 \mathrm{MHz}\right) \delta: 7.75(\mathrm{~d}$, $J=6.0 \mathrm{~Hz}, 1 \mathrm{H}), 6.96$ (d, $J=8.0 \mathrm{~Hz}, 1 \mathrm{H}), 6.89$ (d, $J=2.0$ $\mathrm{Hz}, 1 \mathrm{H}), 6.82(\mathrm{~d}, J=8.0 \mathrm{~Hz}, 2 \mathrm{H}), 6.75(\mathrm{dd}, J=8.0,10.0$ $\mathrm{Hz}, 1 \mathrm{H}), 6.68(\mathrm{~d}, J=1.5 \mathrm{~Hz}, 1 \mathrm{H}), 6.62(\mathrm{dd}, J=8.0,9.5 \mathrm{~Hz}$, $1 \mathrm{H}), 4.13$ (dd, $J=9.0,16.5 \mathrm{~Hz}, 1 \mathrm{H}), 3.91$ (dd, $J=8.5,17.0$ $\mathrm{Hz}, 1 \mathrm{H}), 3.69$ (d, $J=1.5 \mathrm{~Hz}, 6 \mathrm{H}), 3.64(\mathrm{~s}, 3 \mathrm{H}), 2.93 \sim 2.88$ (m, 2H), 2.84 2.75 (m, 4H); ESI-MS $m / z: 466[\mathrm{M}+1]^{+}$.

(3R,4R)-3-[3-甲氧基-4-(2-O-4-三氟甲基-嘧啶基)苯甲基]-4-(3,4-二甲氧基-苯甲基)-丁内酯(11j): $0.2 \mathrm{~g}$ 浅 棕色油状液体, 产率 $75 \% .{ }^{1} \mathrm{H} \mathrm{NMR}\left(\mathrm{CDCl}_{3}, 500 \mathrm{MHz}\right) \delta$ : $8.71(\mathrm{~d}, J=5.0 \mathrm{~Hz}, 1 \mathrm{H}), 7.33$ (d, $J=4.5 \mathrm{~Hz}, 1 \mathrm{H}), 7.11$ (d, $J=8.0 \mathrm{~Hz}, 1 \mathrm{H}), 6.81(\mathrm{~d}, J=2.0 \mathrm{~Hz}, 1 \mathrm{H}), 6.77 \sim 6.73(\mathrm{~m}$, $2 \mathrm{H}), 6.56 \sim 6.54(\mathrm{~m}, 1 \mathrm{H}), 6.52(\mathrm{~d}, J=2.0 \mathrm{~Hz}, 1 \mathrm{H}), 4.19$ (dd, $J=9.5,16.5 \mathrm{~Hz}, 1 \mathrm{H}), 3.91$ (dd, $J=9.0,17.0 \mathrm{~Hz}, 1 \mathrm{H}$ ), $3.85(\mathrm{~s}, 3 \mathrm{H}), 3.82(\mathrm{~s}, 3 \mathrm{H}), 3.68(\mathrm{~s}, 3 \mathrm{H}), 3.06 \sim 2.96(\mathrm{~m}$, $2 \mathrm{H}), 2.69 \sim 2.51(\mathrm{~m}, 4 \mathrm{H})$; ESI-MS $m / z: 519[\mathrm{M}+1]^{+}$.

(3R,4R)-3-[3-甲氧基-4-(2-O-4,6-二甲氧基-嘧啶基)苯甲基]-4-(3,4-二甲氧基-苯甲基)-丁内酯(11k)：0.18 g 浅棕色粉末, 产率 $66.3 \% .{ }^{1} \mathrm{H} \mathrm{NMR}\left(\mathrm{CDCl}_{3}, 500 \mathrm{MHz}\right) \delta$ : 7.07 (d, $J=8.0 \mathrm{~Hz}, 1 \mathrm{H}), 6.80(\mathrm{~d}, J=2.0 \mathrm{~Hz}, 1 \mathrm{H}), 6.75 \sim$ 
$6.73(\mathrm{~m}, 2 \mathrm{H}), 6.53 \sim 6.50(\mathrm{~m}, 4 \mathrm{H}), 5.73(\mathrm{~s}, 1 \mathrm{H}), 4.10(\mathrm{dd}$, $J=9.5,16.5 \mathrm{~Hz}, 1 \mathrm{H}), 3.87$ (dd, $J=9.0,17.0 \mathrm{~Hz}, 1 \mathrm{H}), 3.84$ $(\mathrm{s}, 3 \mathrm{H}), 3.82(\mathrm{~s}, 3 \mathrm{H}), 3.73(\mathrm{~s}, 3 \mathrm{H}), 3.72(\mathrm{~s}, 6 \mathrm{H}), 2.96 \sim 2.92$ $(\mathrm{m}, 2 \mathrm{H}), 2.63 \sim 2.44(\mathrm{~m}, 4 \mathrm{H})$; ESI-MS m/z: $511[\mathrm{M}+1]^{+}$.

\section{References}

[1] Fu, L.-C; Xu, P.-P; Liu, N; Yang, Z.-F.; Zhang, F.-X.; Hu, Y.-J. Trad. Chin. Drug Res. Clin. Pharmacol. 2008, 4, 266 (in Chinese). (符林春, 徐培平, 刘妮, 杨子峰, 张奉学, 胡英杰, 中药新药与 临床药理, 2008, 4, 266.)

(b) Fischer, J.; Reynolds, A.-J.; Sharp, L.-A. Org. Lett. 2004, 6, 1345.

(c) Mi, J.-Y.; Song, C.-Q. Lishizhen Medicine Materia Medica Res.
2002, 13, 168 (in Chinese)

(米靖宇, 宋纯清, 时珍国医国药, 2002, 13, 168.)

(d) Wang, L.; Zhao, F.; Liu, K. Chin. Trad. Herb. Drugs 2008, 39, 467 (in Chinese).

(王潞，赵烽，刘珂，中草药, 2008, 39, 467.)

[2] (a) Stephen, W.-W.; Hallstrom, K.-N. J. Org. Chem. 2006, 71, 1080.

(b) Colombo, M.; Giglio, M.; Peretto, I. J. Heterocycl. Chem. 2008, 45, 1077.

[3] Tibiletti, F.; Simonetti, M.; Nicholas, K.-M.; Palmisano, G.; Parravicini, M.; Imbesi, F.; Tollari, S.; Penoni, A. Tetrahedron 2010, 66, 1280.

[4] Shi, J.; Manolikakes, G.; Yeh, C. J. Am. Chem. Soc. 2011, 133, 8014. 